\title{
PAPER
}

\section{Acoustic characteristics of Wadaiko (traditional Japanese drum) with wood plastic shell}

\author{
Teruaki Ono ${ }^{1}$, Isoko Takahashi ${ }^{2}$, Yasuo Takasu ${ }^{2}$, \\ Yaichi Miura $^{3}$ and Ugai Watanabe ${ }^{4, *}$ \\ ${ }^{1}$ Faculty of Engineering, Gifu University, \\ 1-1 Yanagido, Gifu, 501-1193 Japan \\ ${ }^{2}$ Aichi Industrial Technology Institute, \\ Nishishinwari, Itsuki-cho, Kariya, 448-0003 Japan \\ ${ }^{3}$ Miura Taikoten, \\ Sugimoto, Rokku-cho, Okazaki, 444-0072 Japan \\ ${ }^{4}$ Faculty of Engineering, Chiba Institute of Technology, \\ 2-17-1 Tsudanuma, Narashino, 275-0016 Japan
}

( Received 7 November 2008, Accepted for publication 15 May 2009)

\begin{abstract}
Keyaki wood is the most suitable material for the "Wadaiko, a traditional Japanese drum." However, the amount of wood with excellent quality available for producing the wadaiko has decreased in recent years. Wadaiko shells made of artificial materials are an effective substitute for the traditional wooden wadaiko. In this study, prototypes of the wadaiko were made using a wood plastic and their sound characteristics were examined. The vibrational properties of the wood plastic were investigated and compared with those of keyaki and sen woods used for the traditional wooden wadaiko. The sound characteristics of the wood plastic wadaiko were more complex than those of the keyaki wadaiko. The Chladni experiment on the wood plastic wadaiko clarified that the respective modal patterns appeared at two or three resonant frequencies with increasing the degree of the modes. The sound components of the wood plastic shell did not affect the overall sound characteristics of the wadaiko. The internal friction of wood plastic was larger than those of keyaki and sen, whereas the specific Young's modulus in the grain direction was smaller than those of keyaki and sen. The results of this study indicated that the mechanical properties of wadaiko shell materials strongly affected both the tension of the membrane and the boundary condition between the membrane and shell. A large specific Young's modulus as well as high strength comparable to that of keyaki is necessary for wood plastics to be used as a substitute material for the wadaiko shell.
\end{abstract}

Keywords: Japanese drum, Wood plastics, Sound characteristics, Chladni experiment

PACS number: 43.75.Hi [doi:10.1250/ast.30.410]

\section{INTRODUCTION}

Drums are the oldest musical instruments in human history. The important role of drums is to provide rhythm in musical pieces. Also, drums are an essential tool in religious rites [1]. The sounds of drums are deeply linked with the human psyche. In Japan, traditional wooden drums called Wadaiko are made. Various types of wadaiko often appear in traditional public entertainment such as gagaku (imperial court music) and Noh plays.

The sound characteristics of drums strongly depend on the shapes of their shells, the tension of the membranes and

*e-mail: ugai@sun.it-chiba.ac.jp the qualities of the materials used for the shells and membranes. The former two factors can be changed during manufacturing, but it is almost impossible to control the quality of shell materials. The most suitable shell material for the wadaiko is keyaki (Zelkova serrata), which is one of the famous hardwoods of East Asia. Other hardwoods such as sen (Kalopanax pictus) and tochi (Aesculuc turbinata) are also used for the wadaiko shell. The shells are made by hollowing out the logs of these hardwood species. These woods exhibit the physical properties required for the wadaiko and have a beautiful and characteristic grain pattern on the tangential surfaces. However, the amount of wood with suitable quality available for producing the wadaiko shell has decreased in recent years. 
Cow skin membranes stretched with high tension are tightly attached to both ends of the wadaiko shell by rivets or ropes. Therefore, the shell materials used for the wadaiko should have sufficient strength to support the stretched membranes. Generally, many hardwoods exhibit high mechanical properties because of their large densities [2]. The effects of the vibrational properties of the shell material on the sound characteristics of the wadaiko have never been clarified. It is essential to determine the vibrational properties of wood used for the wadaiko shell, so that a substitute wood can be found to replace the traditional shell materials. Furthermore, the vibrational properties will be an important criterion when developing substitute materials for the wood shell. Artificial materials that mimic the structural and physical characteristics of wood can contribute to resolving the problem of the scarcity of suitable materials for musical instruments $[3,4]$.

In this study, the effectiveness of a wood plastic for the shell material of the wadaiko was investigated. Prototypes of the wood plastic wadaiko were made by Aichi Industrial Technology Institute and Miura Taikoten Co., Ltd. Their sound characteristics were examined and compared with those of a traditional wadaiko made of keyaki. The vibrational properties of the wood plastic were measured and compared with those of plainsawn wood of keyaki and sen woods traditionally used for the wadaiko. On the basis of the experimental results, the suitability of using the wood plastic in the wadaiko is discussed.

\section{TESTED WADAIKO AND MEASURING METHODS}

\subsection{Prototypes of Wood Plastic Wadaiko and Keyaki Wadaiko}

The wood plastic was produced as follows: Planer shavings of maple (Acer mono) were steamed at 160 to $200^{\circ} \mathrm{C}$ for 20 minutes, air-dried, and then milled to obtain a powder. This powder was pressed in specially designed molds for making wood plastic shells. The pressure and temperature during pressing were set to $30 \mathrm{MPa}$ and $180^{\circ} \mathrm{C}$, respectively [5]. The thickness of the shell was $20 \mathrm{~mm}$ and its density $\rho$ was $1.44 \mathrm{~g} / \mathrm{cm}^{3}$.

Two prototypes of the wadaiko were made. In the wood plastic wadaiko with a barrel-like shell body (b-wadaiko, center of Fig. 1), two cow skin membranes were attached to the shell using rivets, whereas in the wood plastic wadaiko with a pail-like shell body (p-wadaiko, right of Fig. 1), the two membranes were attached using ropes. Note that part of the shell of the b-wadaiko was damaged during the tacking down of the membranes with rivets because of the hardness of the wood plastic.

To compare the sound characteristics of the wood plastic wadaiko, a keyaki wadaiko, whose two cow skin
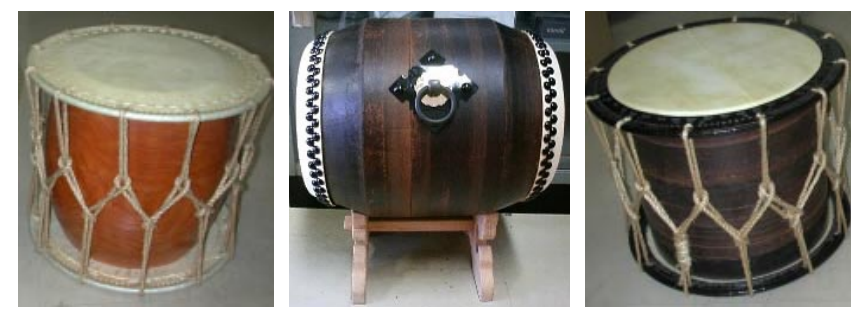

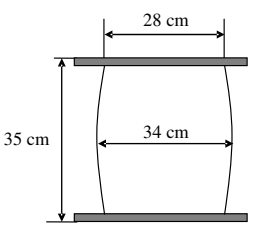

Keyaki Wadaiko

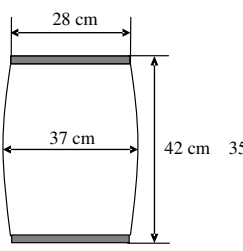

b-Wadaiko

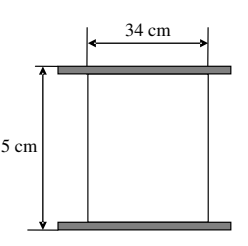

p-Wadaiko
Fig. 1 Exterior views and dimensions of keyaki wadaiko, b-wadaiko (wood plastic wadaiko with a barrel-like shell body) and p-wadaiko (wood plastic wadaiko with a pail-like shell body).

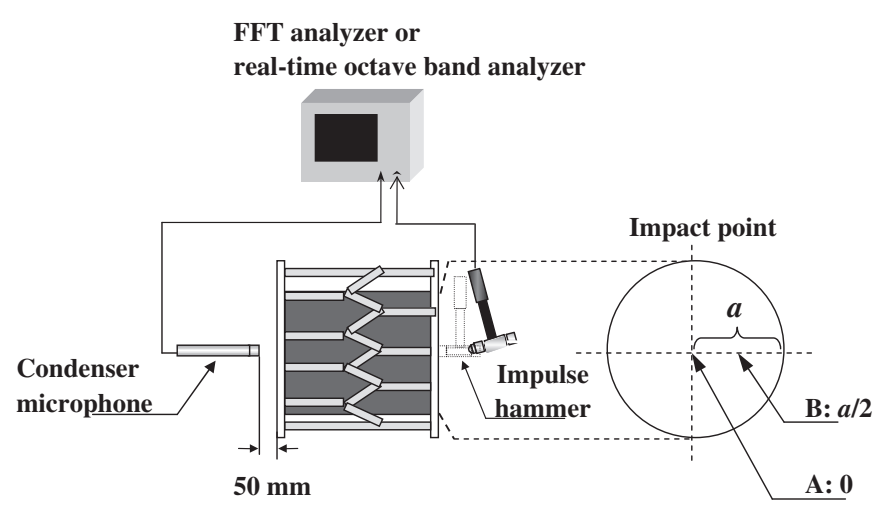

Fig. 2 Apparatus for measuring the sound characteristics of the wadaiko by tapping.

membranes were attached using ropes, with a barrel-like shell was prepared (left of Fig. 1).

The thickness of the cow skins used as the membranes of the wadaiko was measured using a digital caliper. The $\rho$ of the skin was measured by the gas phase substitution method.

\subsection{Sound Characteristics of the $\mathbf{P}$ - and B-wadaikos and Keyaki Wadaiko}

The sound characteristics of the p-wadaiko and bwadaiko were investigated by a tapping method and were compared with those of a traditional wadaiko made of keyaki. One side membrane was hit with an impulse hammer and the sound was monitored by a condenser microphone set up in front of the other membrane (Fig. 2). The points where the membrane was hit were the center (point A) and half the radius away from the center (point B). The microphone was set up $50 \mathrm{~mm}$ away from the center of the membrane. Both the tapping and sound 
signals were input into an FFT analyzer to obtain their transfer functions. The window function was rectangular and the sampling number was 1,024 . The data lengths were $40 \mathrm{~ms}$ for the frequency range from 0 to $10 \mathrm{kHz}$ and $200 \mathrm{~ms}$ for the frequency range from 0 to $2 \mathrm{kHz}$.

\subsection{Modal Patterns of the P-wadaiko and Barrel-like Keyaki Wadaiko}

The frequency characteristics of the keyaki wadaiko and p-wadaiko were investigated by the Chladni method. Sand with a particle diameter of about $0.6 \mathrm{~mm}$ was spread on one membrane and then the other membrane was excited using a loudspeaker while manually sweeping the frequency. The modal patterns were captured using a digital camera.

\subsection{Sound Characteristics of the P-wadaiko Shell and Barrel-like Keyaki Wadaiko Shell}

For the p-wadaiko and keyaki wadaiko, the sound characteristics of their shells were measured to investigate their effects on the whole sound characteristics. The center of the wadaiko shell in the axial direction was hit with an impulse hammer and the relative sound pressure level was analyzed by the same method as that illustrated in Fig. 2. The same measurements were then conducted after the membranes were detached from the shells.

\subsection{Frequency Responses and Vibrational Properties of the Shell Materials}

To investigate the frequency responses and vibrational properties of the shell materials of the traditional wooden wadaiko, plainsawn wood samples of keyaki and sen were prepared. The densities $\rho$ of keyaki and sen were 0.681 and $0.542 \mathrm{~g} / \mathrm{cm}^{3}$, respectively. Square specimens of 105 $\mathrm{mm}(L) \times 105 \mathrm{~mm}(T) \times 3.0 \mathrm{~mm}(R)$ were cut from both samples, where $L$ denotes the direction of the grains and $T$ denotes the tangential direction with respect to the tree rings. The frequency response characteristics of the specimens were measured by the forced vibration method [6]. The center of the plate was excited by a sine wave sweeping the frequency range of 0.1 to $10 \mathrm{kHz}$ and the vibrational response was detected using a condenser microphone. The Young's moduli and internal frictions of the square specimens in the $L$ and $T$ directions, $E_{L}, E_{T}$, $Q_{L}^{-1}$ and $Q_{T}^{-1}$, respectively, were measured by a free-free flexural vibration method.

Plate specimens of the wood plastic were made to evaluate their mechanical properties. The wood powder, treated as previously described, was pressed in a mold with an area of $105 \mathrm{~mm} \times 105 \mathrm{~mm}$. The pressing conditions were the same as those for the wood plastic shell. Square specimens of $105 \mathrm{~mm} \times 105 \mathrm{~mm} \times 3.0 \mathrm{~mm}$ were cut from the plate specimens to investigate their frequency respons-
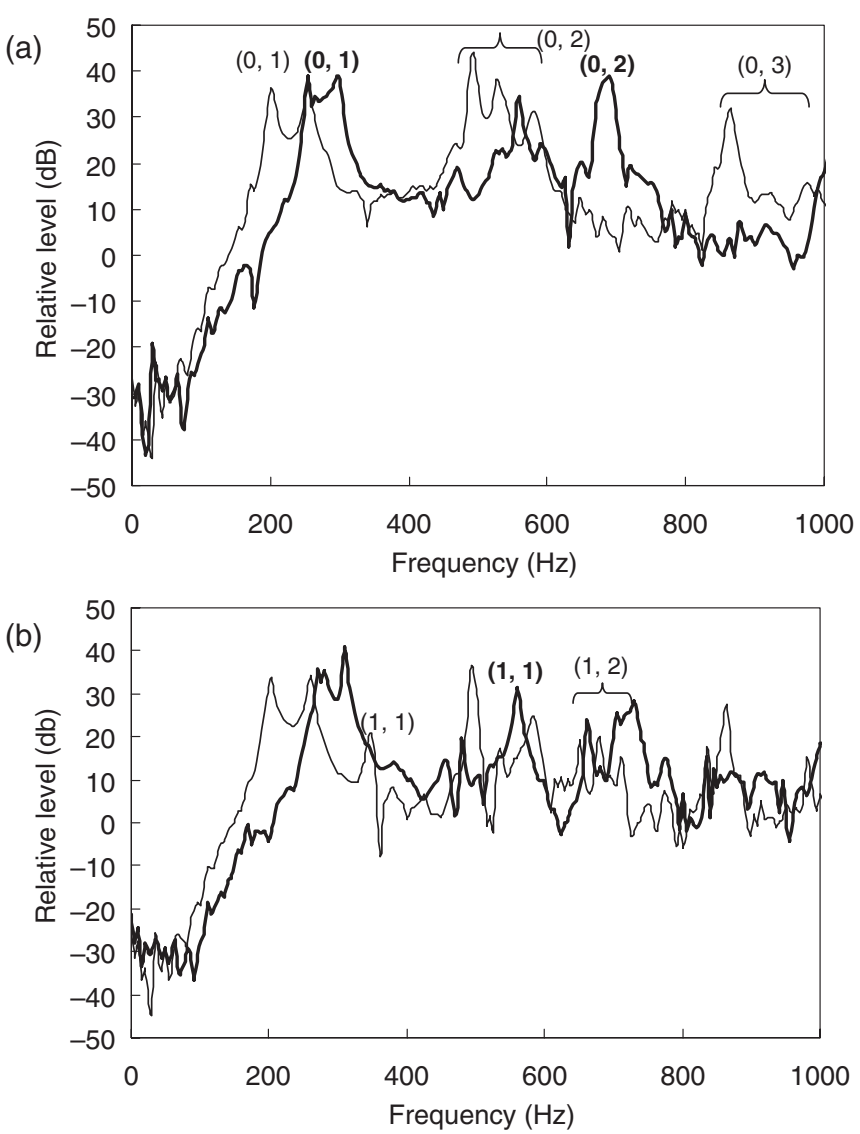

Fig. 3 Sound pressure spectra of keyaki wadaiko (thick line) and p-wadaiko (fine line): (a) spectra for point A, (b) spectra for point $\mathrm{B}$.

es by the forced vibration method. Rectangular specimens of $15.5 \mathrm{~mm} \times 3.0 \mathrm{~mm} \times 150 \mathrm{~mm}$ were also cut, for which $E$ and $Q^{-1}$ were measured by the free-free flexural vibration method.

\section{RESULTS AND DISCUSSION}

\subsection{Sound Pressure Spectra of the P-and B-wadaikos and Keyaki Wadaiko}

Figure 3 shows the sound pressure spectra of the keyaki wadaiko and p-wadaiko. In Fig. 3(a), the spectra at point A are compared between the two types of wadaiko, and those at point B are compared in Fig. 3(b). For point A, clear peaks were observed at 255, 295, 560 and $690 \mathrm{~Hz}$ in the spectrum of the keyaki wadaiko, whereas in the spectrum of the p-wadaiko, peaks were observed at 200, 255, 495, 525, 580 and $865 \mathrm{~Hz}$. For point B, clear peaks were observed at 270, 310, 485, 560, 660 and $730 \mathrm{~Hz}$ in the spectrum of the keyaki wadaiko, whereas in the spectrum of the p-wadaiko, peaks were observed at 205, 260, 350, $495,585,650,680,710,835$ and $865 \mathrm{~Hz}$. Figure 4 shows a comparison of the sound pressure spectra between the keyaki wadaiko and the b-wadaiko. The spectrum at point A for the b-wadaiko exhibited clear peaks at 215, 245, 490, 585880,915 and $960 \mathrm{~Hz}$. For point B, peaks were observed 

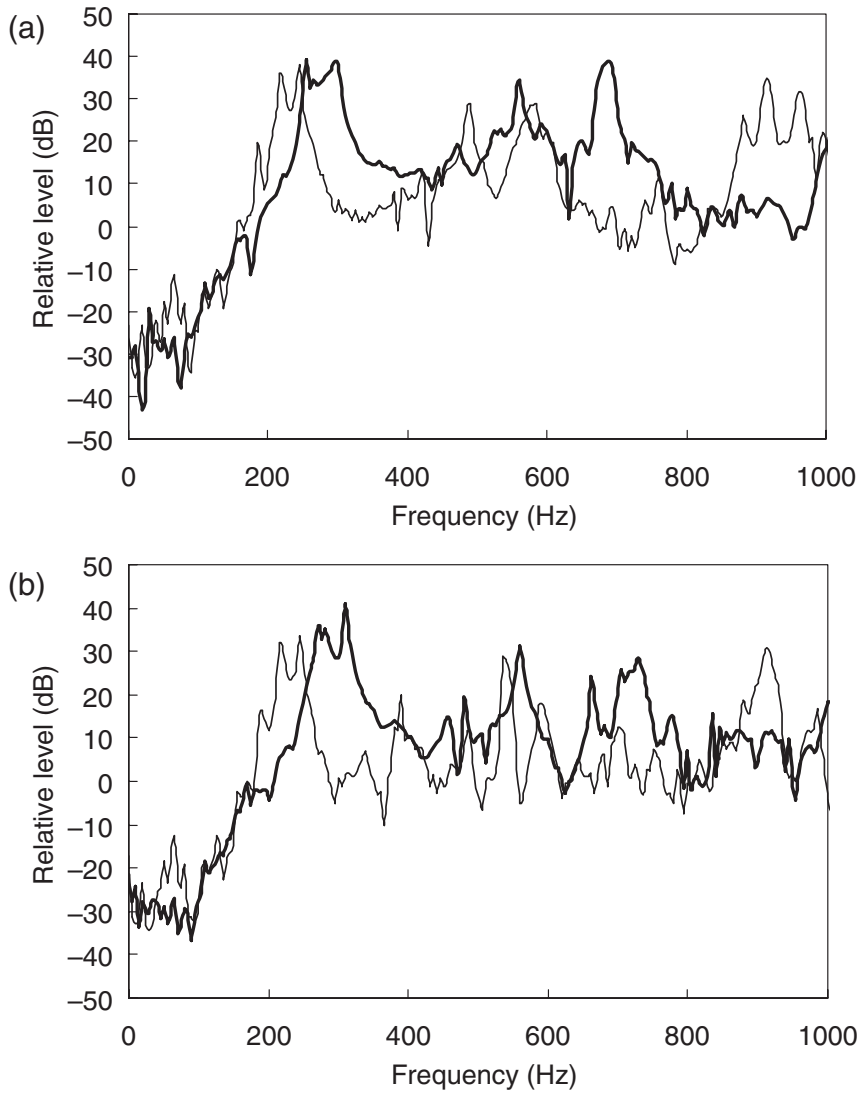

Fig. 4 Sound pressure spectra of keyaki wadaiko (thick line) and b-wadaiko (fine line): (a) spectra for point A,

(b) spectra for point $\mathrm{B}$.

at $215,245,390,535,590,915$ and $985 \mathrm{~Hz}$. Although the shell shape of the b-wadaiko was close to that of the keyaki wadaiko, the spectrum exhibited more peaks. According to these results, the sounds of the wood plastic wadaiko included more components than those of the keyaki wadaiko, resulting in more complex sound characteristics.

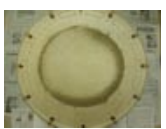

$(0,1) 323 \mathrm{~Hz}$
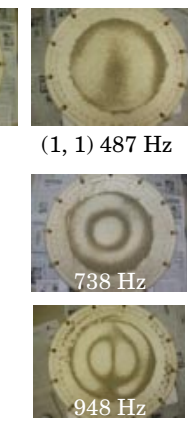

$(1,1) 487 \mathrm{~Hz}$

$(1,2)$

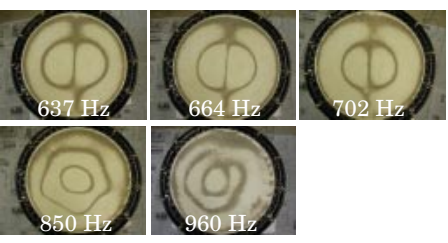

Keyaki Wadaiko $(\mathbf{0 , 2})$
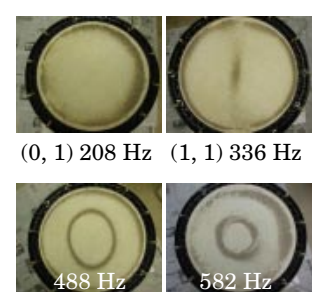

$(0,1) 208 \mathrm{~Hz} \quad(1,1) 336 \mathrm{~Hz}$

$(\mathbf{0 , 3 )}$

p-Wadaiko
Fig. 5 Modal patterns of keyaki wadaiko and p-wadaiko.

\subsection{Vibrational Mode of Membranes Attached to the P-wadaiko and Keyaki Wadaiko}

Figure 5 shows the modal frequencies of the membranes attached to the keyaki wadaiko and p-wadaiko. In Table 1, the frequencies at which the respective modal patterns appeared are compared with those in the sound pressure spectra shown in Fig. 3. The frequency ratios of the respective modes $(m, n)$ to the frequency of the $(0,1)$ mode $f_{m n} / f_{01}$ are also compared with their theoretical values for an ideal circular membrane.

In the keyaki wadaiko, the peak at $295 \mathrm{~Hz}$ in the spectrum shown in Fig. 3(a) was assigned to the oscillation of the $(0,1)$ mode. The $(1,1),(0,2)$ and $(1,2)$ modal patterns were observed at their respective single frequencies. The peak at $690 \mathrm{~Hz}$ for point $\mathrm{A}$ was assigned to the oscillation of the $(0,2)$ mode. The peak at $560 \mathrm{~Hz}$ in the spectrum shown in Fig. 3(b) was assigned to the oscillation of the $(1,1)$ mode. The peak corresponding to the

Table 1 Assignment of peaks on sound pressure spectra to vibrational modes of membrane.

\begin{tabular}{|c|c|c|c|c|c|c|}
\hline Wadaiko & mode $(m, n)$ & $f_{\mathrm{Ch}}(\mathrm{Hz})$ & $f_{\mathrm{p}}(\mathrm{Hz})$ & $\left(f_{m n} / f_{01}\right)_{\mathrm{th}}$ & $\left(f_{m n} / f_{01}\right)_{\mathrm{Ch}}$ & $\left(f_{m n} / f_{01}\right)_{\mathrm{p}}$ \\
\hline Keyaki & $(0,1)$ & 323 & 295 & 1.00 & 1.00 & 1.00 \\
\hline \multirow[t]{3}{*}{ Wadaiko } & $(1,1)$ & 487 & 560 & 1.59 & 1.51 & 1.90 \\
\hline & $(0,2)$ & 738 & 690 & 2.30 & 2.28 & 2.34 \\
\hline & $(1,2)$ & 948 & 1015 & 2.91 & 2.93 & 3.44 \\
\hline \multirow[t]{10}{*}{ P-wadaiko } & $(0,1)$ & 208 & 200 & 1.00 & 1.00 & 1.00 \\
\hline & $(1,1)$ & 336 & 350 & 1.59 & 1.62 & 1.75 \\
\hline & $(0,2)$ & 488 & 495 & 2.30 & 2.35 & 2.48 \\
\hline & & & 525 & & & 2.63 \\
\hline & & 582 & 580 & 2.30 & 2.80 & 2.90 \\
\hline & $(1,2)$ & 637 & 650 & 2.91 & 3.06 & 3.25 \\
\hline & & 664 & 680 & 2.91 & 3.19 & 3.40 \\
\hline & & 702 & 710 & 2.91 & 3.38 & 3.55 \\
\hline & $(0,3)$ & 850 & 865 & 3.60 & 4.09 & 4.33 \\
\hline & & 960 & 975 & 3.60 & 4.62 & 4.88 \\
\hline
\end{tabular}

Ch: data obtained by the Chladni method, p: data obtained by a tapping method, th: theoretical values 
oscillation of the $(1,2)$ mode appeared at $1015 \mathrm{~Hz}$ in the same spectrum. All values of $f_{m n} / f_{01}$ for the modal patterns $\left(f_{m n} / f_{01}\right)_{\mathrm{Ch}}$ almost coincided with the theoretical values, whereas the values of $f_{m n} / f_{01}$ obtained from the frequencies of the spectrum peaks $\left(f_{m n} / f_{01}\right)_{\mathrm{p}}$ were larger than the theoretical values. This was attributed to the effect of air loading on the membranes. Theoretical analysis of the effect of air loading on the modal frequencies of a membrane used in timpani clarified that the value of $f_{m n} / f_{11}$ with a kettle was larger than that without a kettle and the ideal value for all modes [1]. The result in Table 1 was similar to that in [1]. Furthermore, the frequencies of $(1, n)$ modes were lower than those of the corresponding peaks. This was considered to result from the suppression of the oscillation by the mass of sand on the node line across the membrane.

In the p-wadaiko, the $(0,1)$ modal pattern appeared at $208 \mathrm{~Hz}$; therefore, the peak at $200 \mathrm{~Hz}$ in the spectrum shown in Fig. 3(a) was assigned to the oscillation of this mode. The $(1,1)$ modal pattern was observed at $336 \mathrm{~Hz}$; thus, the peak at $350 \mathrm{~Hz}$ in the spectrum shown in Fig. 3(b) was assigned to the oscillation of this mode. The $(0,2)$ modal pattern appeared at the two frequencies of 488 and $582 \mathrm{~Hz}$. The three peaks in the frequency range from 495 to $580 \mathrm{~Hz}$ for point $\mathrm{A}$ were assigned to the oscillation of this mode. This modal pattern could only be detected at the two modal frequencies by the Chladni method because of its low frequency resolution. The three peaks in the frequency range from 650 to $710 \mathrm{~Hz}$ for point $\mathrm{B}$ were assigned to the oscillation of the $(1,2)$ mode. The two peaks at 865 and $975 \mathrm{~Hz}$ for point A were assigned to the oscillation of the $(0,3)$ mode. The values of $\left(f_{m n} / f_{01}\right)_{\mathrm{p}}$ were larger than the theoretical values, as for the case of the keyaki wadaiko.

The peaks assigned to the oscillation modes of the membrane are indicated in Figs. 3(a) and 3(b). For both the keyaki wadaiko and the p-wadaiko, almost all sound components originated from the vibration of the membrane attached to the shell. A clear peak was observed at $255 \mathrm{~Hz}$ in both spectra in Fig. 3(a). The spectra in Fig. 3(b) exhibit clear peaks at a frequency from 260 to $270 \mathrm{~Hz}$. As shown in Fig. 1, the inner volume of the shell was almost the same for the keyaki wadaiko and the p-wadaiko. Therefore, these peaks were considered to correspond to a sound component depending on the internal volume of the shell.

\subsection{Sound Pressure Spectra of the P-wadaiko Shell and Keyaki Wadaiko Shell}

In Fig. 6(a), the sound pressure spectrum of the keyaki wadaiko shell with membranes is compared with those of the keyaki wadaiko. The spectrum of the shell without membranes is similarly compared in Fig. 6(b). A peak was detected at $360 \mathrm{~Hz}$ in the spectrum of the shell that did not correspond to any peaks in the overall sound pressure
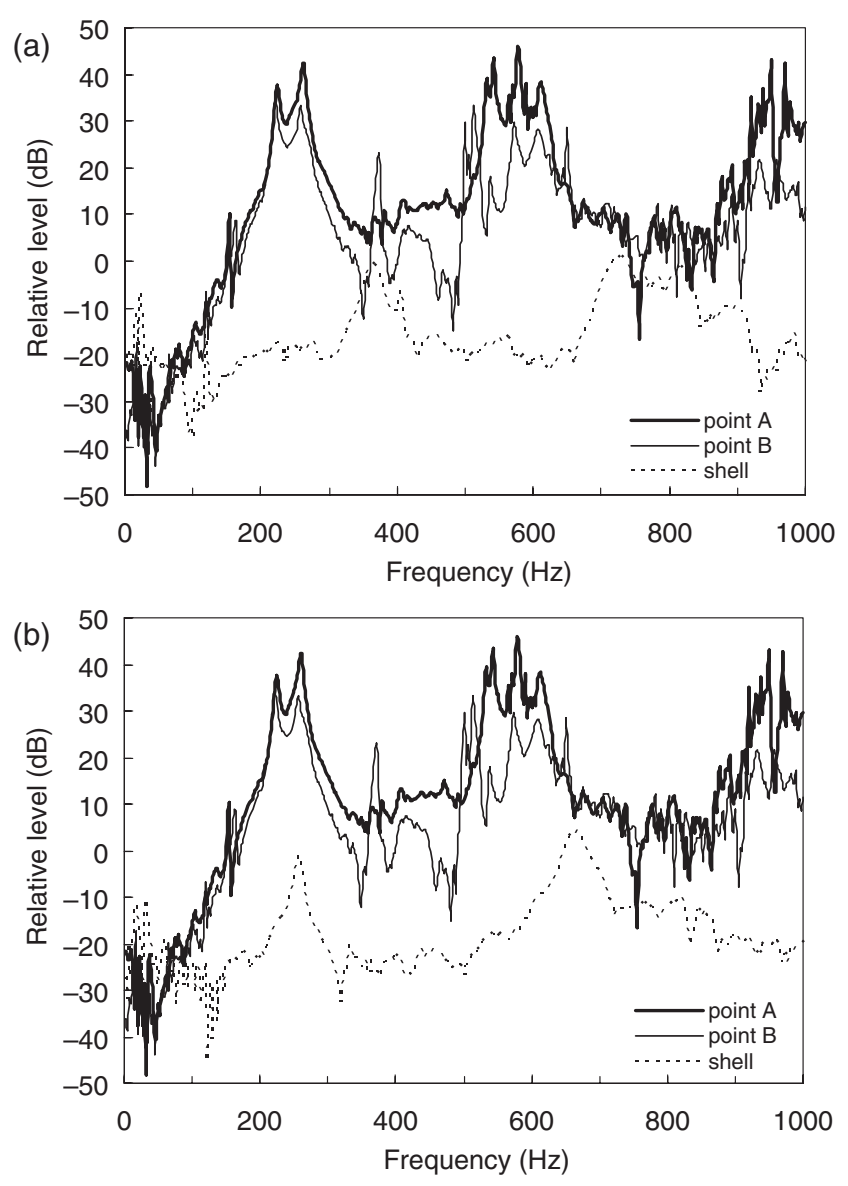

Fig. 6 Relationship between overall sound pressure spectra and spectrum of the shell in the keyaki wadaiko: (a) with membranes, (b) without membranes.

spectra. This peak shifted markedly to $260 \mathrm{~Hz}$ after the membranes were detached from the shell, as shown in Fig. 6(b). Figure 7 shows the sound pressure spectra for the p-wadaiko shell. A peak was detected at $325 \mathrm{~Hz}$ in the spectrum for the shell with membranes that did not correspond to any peaks in the overall sound pressure spectra (Fig. 7(a)). This peak shifted slightly to $295 \mathrm{~Hz}$ after the membranes were detached from the shell (Fig. 7(b)). These results clarified that the sound components of the shell did not affect the whole sound characteristics of the wadaiko. According to the results for the keyaki wadaiko, the tension of the membranes affected the sound components of the wadaiko shell, which were considered to depend on the mechanical properties of the shell materials.

\subsection{Physical Properties of Shell Materials and Mem- brane of the Wadaiko}

Figure 8 shows the frequency response characteristics of square plates of keyaki, sen and wood plastic. The peaks appearing in the frequency range from 250 to $500 \mathrm{~Hz}$ were assigned to the proper oscillation of wood specimens in the $T$ direction, which coincides with the circumferential 
(a)

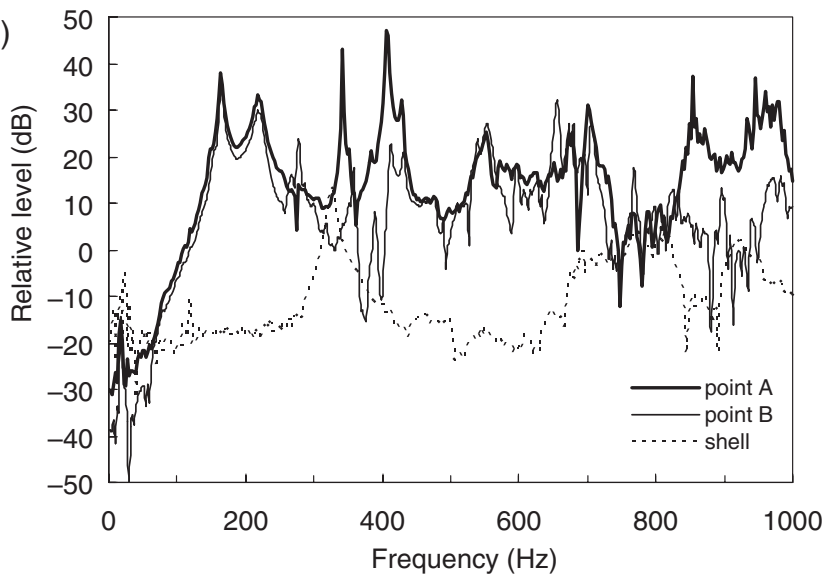

(b)

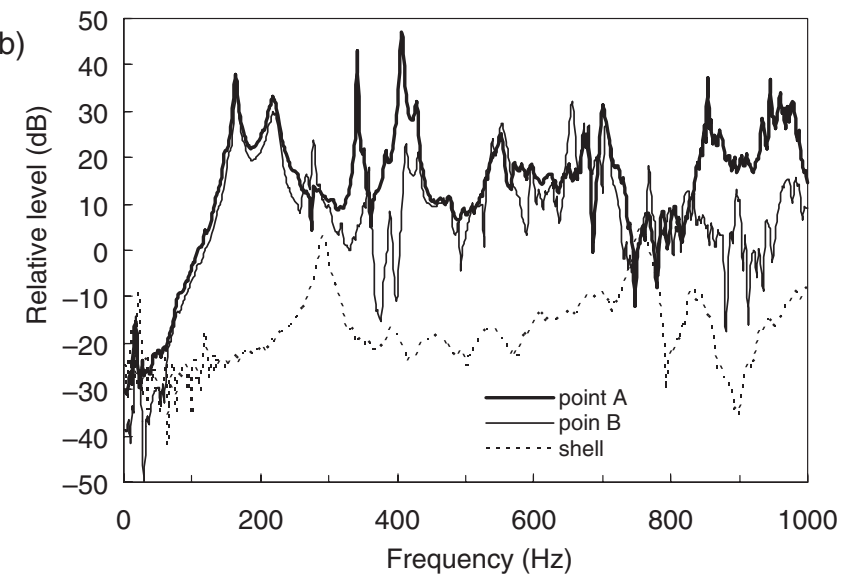

Fig. 7 Relationship between overall sound pressure spectra and the spectrum of the shell in the p-wadaiko: (a) with membranes, (b) without membranes.

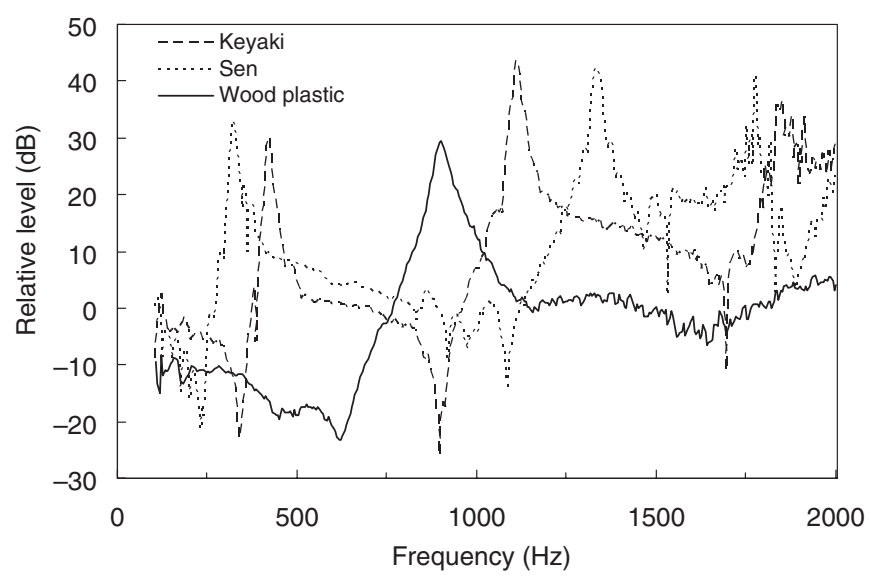

Fig. 8 Sound pressure spectra of square specimens of keyaki, sen and wood plastic measured by forced vibration method.

direction of the wadaiko shell. The peaks appearing in the frequency range from 1,000 to $1,500 \mathrm{~Hz}$ were assigned to the proper oscillation in the $L$ direction, which coincides with the axial direction of the wadaiko shell. Fine peaks were observed at frequencies over $1,700 \mathrm{~Hz}$ for both the keyaki and sen samples. On the other hand, a clear single peak was detected at $905 \mathrm{~Hz}$ for wood plastic plate, indicating that the wood plastic exhibited isotropic elasticity.

Table 2 shows the Young's modulus $E$ and internal friction $Q^{-1}$ of keyaki, sen and wood plastic. The resonant frequencies $f$ are also shown in the table. For the wood plastic, $E$ and $Q^{-1}$ for the rectangular specimen are shown in the table. $E_{L}$ for the wood plastic was the largest among the three different wadaiko shell materials. The elastic anisotropies $E_{L} / E_{T}$ of keyaki and sen were 6.83 and 15.0, respectively. $E_{L} / E_{T}$ for the wood plastic was 1.00 . The specific Young's modulus $E_{L} / \rho$ of the wood plastic plate was the smallest among the three wadaiko shell materials, and $E_{L} / \rho$ for sen was the largest. $Q_{L}^{-1}$ for the wood plastic was three times larger than those of keyaki and sen.

The thickness of the cow skin membranes was 1.0 to $1.5 \mathrm{~mm}$. The density $\rho$, measured by the gas-phase substitution method, was $1.38 \mathrm{~g} / \mathrm{cm}^{3}$.

\subsection{Complex Sound Characteristics of the Wood Plastic Wadaiko and the Mechanical Properties of Wood Plastic}

The sound characteristics of the two types of wood plastic wadaiko were more complex than those of the traditionally used keyaki wadaiko. This did not result from their shell shapes. Almost all the peaks in the sound pressure spectra of the p-wadaiko could be assigned to the oscillation modes of the membrane, and the sound characteristics of the wadaiko shell did not clearly correspond to the overall characteristics of the wadaiko. From these results, it was considered that the proper oscillation of the shell was not involved in the complex sound characteristics of the wood plastic wadaiko. Therefore, the mechanical properties of the wood plastic are considered to affect the complex sound characteristics of the wood plastic wadaiko.

For the keyaki wadaiko, the peaks in the sound pressure spectrum of the shell markedly shifted to lower frequencies when the membranes were detached from the shell. This may be attributed to the release of the deformation caused by the high tension of the membranes. On the other hand, a slight downward shift in frequency was detected for the pwadaiko shell (Fig. 7). The shell of the keyaki wadaiko can elastically deform during the attachment of the membranes with high tension because of its large specific elastic modulus $E / \rho$. During this process, the circumference of the membranes may be ideally fixed with the shell. Therefore, the respective modal patterns can appear at specific frequencies. The Young's modulus of the wood plastic used in this experiment was larger than those of keyaki and sen owing to its high density (Table 2). The shell of the wood plastic wadaiko is not deformable when the same 
Table 2 Density and vibrational properties of square specimens of keyaki, sen and wood plastic.

\begin{tabular}{cccccccccc}
\hline \multirow{2}{*}{ Specimens } & $\begin{array}{c}\rho \\
\mathrm{g} / \mathrm{cm}^{3}\end{array}$ & $\begin{array}{c}f_{L} \\
\mathrm{~Hz}\end{array}$ & $\begin{array}{c}E_{L} \\
\mathrm{GPa}\end{array}$ & $\begin{array}{c}Q_{L}^{-1} \\
\times 10^{-3}\end{array}$ & $\begin{array}{c}f_{T} \\
\mathrm{~Hz}\end{array}$ & $\begin{array}{c}E_{T} \\
\mathrm{GPa}\end{array}$ & $\begin{array}{c}Q_{T}^{-1} \\
\times 10^{-3}\end{array}$ & $\begin{array}{c}E_{L} / \rho \\
(\mathrm{km} / \mathrm{s})^{2}\end{array}$ & $E_{L} / E_{T}$ \\
\hline Keyaki & 0.681 & 1,116 & 9.90 & 7.12 & 427 & 1.45 & 17.5 & 14.5 \\
Sen & 0.542 & 1,352 & 10.5 & 8.95 & 349 & 0.70 & 18.1 & 19.4 \\
Wood plastic & 1.440 & 971 & $14.5^{*}$ & $28.8^{*}$ & 968 & $14.5^{*}$ & $28.8^{*}$ & $10.1^{*}$ & 1.00 \\
\hline
\end{tabular}

*These values were measured using rectangular specimens.

tension of membrane is applied; thus, the circumference of the membrane is inferred not to be fixed but to be supported on the shell. Under the boundary condition of support, the effective radius of the membrane for vibration can easily vary, enabling a specific modal pattern of the membrane to appear at a few frequencies.

The mechanical properties of wadaiko shell materials strongly affect both the tension of the membrane and the boundary condition between the membrane and shell. A high elastic recovery force corresponding to the tension of the membrane as well as high strength is needed for the shell of the wadaiko. A material with a large $E / \rho$ exhibits high elastic deformability. $E / \rho$ for wood plastic is smaller than that of keyaki owing to its high density. Wood used for wooden musical instruments generally has a low specific gravity and a large specific Young's modulus [7]. If a large $E / \rho$ comparable to that of keyaki can be obtained by reducing $\rho$, wood plastic can be used as a substitute material for the wood used in the wadaiko shell.

\section{CONCLUSION}

Prototypes of a wood plastic wadaiko were made and their sound characteristics were compared with those of a traditional keyaki wadaiko. The vibrational properties of the wood plastic were investigated and compared with those of keyaki and sen used for the traditional wooden wadaiko. The sound characteristics of the wood plastic wadaiko were more complex than those of the keyaki wadaiko. In the p-wadaiko, the modal patterns of the membrane for the $(0,2),(1,2)$ and $(0,3)$ modes appeared at a few frequencies. Almost all peaks in the sound pressure spectra of the wadaikos were assigned to the oscillation modes of the membrane. The sound components of the shell did not affect the overall sound characteristics for both the keyaki wadaiko and the p-wadaiko. The specific Young's modulus of the wood plastic is smaller than those of keyaki and sen. In the p-wadaiko, the incomplete fixation of the membranes to the shell was considered to cause the complex oscillation of the membrane, resulting in the complex sound characteristics. The mechanical properties of wadaiko shell materials strongly affected both the tension of the membrane and the boundary condition between the membrane and shell. The specific Young's modulus is a key factor for determining the suitability of wadaiko shell materials. A wood plastic with a large specific Young's modulus and a high strength comparable to those of keyaki may be utilized as a substitute material for the wood used in the wadaiko shell.

\section{ACKNOWLEDGEMENTS}

We would like to express our gratitude to Mr. T. Mitsuya and Ms. K. Yanoo, who are graduates of Faculty of Engineering, Gifu University, for their assistances in the experiment performed in this study, and to Professor. S. Yoshikawa of Kyushu University for his advice on the vibration of shells.

\section{REFERENCES}

[1] N. H. Fletcher and T. D. Rossing, "Drums," in The Physics of Musical Instruments, 2nd ed. (Springer-Verlag, New York, 1998), pp. 583-622.

[2] D. W. Green, J. E. Winandy and D. E. Kretschmann, "Mechanical properties of wood," in Wood Handbook (Forest Products Society, Madison, 1999), 4-1-4-44.

[3] T. Ono, S. Miyakoshi and U. Watanabe, "Acoustic characteristics of unidirectionally fiber-reinforced polyurethane foam composite for musical instrument soundboards," Acoust. Sci. \& Tech., 23, 135-142 (2002).

[4] T. Ono and D. Isomura, "Acoustic characteristics of carbon fiber-reinforced synthetic wood for musical instrument soundboards," Acoust. Sci. \& Tech., 25, 475-477 (2004).

[5] Y. Takasu and I. Takahashi, "Manufacture of self-bonding molding from woody material," APAST, 15, 4-7 (2005) (in Japanese).

[6] T. Ono, "Frequency responses of wood for musical instruments in relation to the vibrational properties," J. Acoust. Soc. Jpn. (E), 17, 183-193 (1996).

[7] T. Ono, "Transient response of wood for musical instruments and its mechanism in vibrational property," J. Acoust. Soc. Jpn. (E), 20, 117-124 (1998). 